\title{
A simple and efficient model for indoor path-loss prediction
}

\author{
Constantino Perez-Vega $\nmid$, Jose Luis García G $\dagger$ and \\ José Miguel López Higuera $\ddagger$
}

\author{
$\dagger$ Microwave and Radiocommunications Group, Communications Engineering \\ Department, University of Cantabria, Santander, Spain \\ ¥ Photonics Engineering Group, Electronic Technology and System Engineering \\ Department, University of Cantabria, Santander, Spain
}

Received 24 January 1997, in final form 13 May 1997, accepted for publication 24 June 1997

\begin{abstract}
A power-law path-loss model for indoor communications at $1.8 \mathrm{GHz}$ is examined. In it, the exponent of the distance is treated as a random variable and its behaviour studied through experiments conducted under various propagation conditions in different buildings. The effects of random human traffic in the propagation channel, as well as those of antenna polarization are also experimentally studied. The statistical behaviour of the exponent and its fitting to several distribution functions, in particular Weibull, Nakagami and gamma ones, are also reported. By using the proposed model, new, simple and efficient instruments can be developed as an aid for the estimation of the power budget of indoor wireless communication systems.
\end{abstract}

\section{Introduction}

Indoor propagation models used for characterizing, either the path-loss [1-4] or the impulse response [5,6] are, in general, site specific and can broadly, be classified into two types: deterministic and statistical. The former require a vast amount of data regarding geometry, building materials in walls and floors, furniture, human traffic and so on, which it is not always possible to obtain in practice. Statistical models, on the other hand, do not usually consider the physical aspects of the channel and thus do not require a detailed knowledge of the geometrical and electromagnetic properties of materials and objects in the environment. They are based on the statistical characterization of the received signal and, in general, require previous channel sounding to obtain basic statistical information. From a computational point of view, they are easier to implement, require less computational effort and are less sensitive to the environmental geometry.

Independently of the type of model, the usual way of characterizing the propagation channel is by means of a discrete linear filter $[7,8]$ whose output, in response to an input $x(t)$, is given by

$$
y(t)=\sum_{k=1}^{N} a_{k} x\left(t-\tau_{k}\right) \mathrm{e}^{\mathrm{j} \theta_{k}}
$$

where $N$ is the number of paths followed by the electromagnetic wave between the transmitter and the receiver. (According to a common definition, the propagation channel does not include the antennae. When they are included, the channel is designated the radio channel.) The signal arriving at the receiver through a path $k$ will be delayed by $\tau_{k}$ seconds and its amplitude and phase will be $a_{k}$ and $\theta_{k}$ respectively. If the input signal is a unit impulse, $\delta(t)$, the output will be the channel impulse response

$$
h(t)=\sum_{k=1}^{N} a_{k} \delta\left(t-\tau_{k}\right) \mathrm{e}^{\mathrm{j} \theta_{k}} .
$$

In order to obtain a complete description of the impulse response of the channel, the behaviour of $a_{k}, \tau_{k}$ and $\theta_{k}$ must be known. From a statistical point of view, these variables are random and each one of them must be characterized by an adequate statistical function or model, based on experimental data.

The phase of the signal, $\theta_{k}$, is assumed to follow a uniform distribution in $(0,2 \pi][9,10]$ for a Raleigh fading condition and a Student $\mathrm{t}$ with two degrees of freedom for Rican fading [11]. The delay, $\tau_{k}$, has been modelled in several forms $[5,6,10]$, one of them being a modified Poisson process $(\Delta-\mathrm{K})$ described in [10].

In this paper only the path-loss behaviour (fading) is investigated. This is directly related to $a_{k}$, and the problem has traditionally been treated considering the fading under two distinct aspects: short-term or fast fading and long-term or slow fading. The former occurs along small distances or short intervals and can be studied through measurements in small or local areas, namely an area that covers a few wavelengths. It is generally accepted that $a_{k}$ follows a Rayleigh distribution if there is no dominant path and a Rice distribution if there is a path through which the signal 
arriving to the receiver is significantly greater than the signal through other paths. For slow fading, the behaviour in a large area, namely an area which includes several local areas under similar propagation conditions, is of interest. The mean values of power or attenuation in local areas provide the information needed to study it and, in general, it is accepted that $a_{k}$ follows a log-normal distribution. As a consequence, to describe the statistical behaviour of fading completely, two different distribution functions are required, or a more complex function which includes both, such as that proposed by Suzuki [12].

Knowledge of the fading behaviour provides a criterion by which to determine the dimension of the power budget for a system that must function in a particular environment and allows the prediction of the coverage area for a given signal-to-noise ratio. For such reasons, from the engineering point of view, it is of prime importance to use reliable models for system design. Among the various models for indoor communications, a very simple particular form of a power-law model to characterize path loss is examined in this paper. In such a model, the descriptive parameter is the exponent of the distance, whose statistical behaviour is studied experimentally in several indoor environments. This parameter has several interesting properties, the most important being that, for the range of distances encountered in indoor communications and under specific propagation conditions, namely line of sight (LOS) or non-line of sight (NLOS), its mean value is fairly constant over large areas. This property suggests that only one distribution function is required to describe the pathloss behaviour.

\section{The theory}

The power received by an isotropic antenna in free space is given by

$$
P_{r}=\frac{P_{R A D}}{d^{2}}\left(\frac{\lambda}{4 \pi}\right)^{2}
$$

in watts, where $P_{R A D}$ is the equivalent isotropic radiated power, $d$ is the distance in metres between the transmitting and receiving antennae and $\lambda$ is the wavelength in metres.

Under propagation conditions other than free space, the effects of the various propagation mechanisms, namely reflection, diffraction and, in general, scattering, as well as the effects of directive antenna gains and their coupling with the environment must be taken into account. However, there is no simple way in which the individual contributions of such mechanisms can be evaluated and, therefore, it is valid to assume that their overall contribution to the path loss can be expressed by means of a propagation-dependent attenuation factor, $\alpha_{P}$, in the form

$$
P_{r}=\frac{P_{R A D} \alpha_{P}}{d^{2}}\left(\frac{\lambda}{4 \pi}\right)^{2} .
$$

Since $\alpha_{P}$ depends on many factors which cannot be evaluated individually, it can be assumed a random variable and the best procedure by which to know its behaviour is by sounding the channel at the frequency of interest. Such sounding will provide samples of received power at different points in a particular environment, from which $\alpha_{P}$ can be calculated. However, an alternative approach is to assume that, under propagation conditions other than free space, the received power does not obey a simple inversesquare law, but rather depends on the distance according to $1 / d^{n}$, where $n$ will be, in general, different from its freespace value $(n=2)$. Thus, equation (4) can be written in the following form:

$$
P_{r}=\frac{P_{R A D}}{d^{n}}\left(\frac{\lambda}{4 \pi}\right)^{2} .
$$

In the above expression, the effects of the various propagation mechanisms are embedded within the value of $n$ instead of $\alpha_{P}$ and, from equations (4) and (5), it can be seen that the attenuation factor and the exponent of distance are related by

$$
\alpha_{P}=\frac{1}{d^{n-2}}
$$

which can be interpreted as the excess attenuation factor referred to free space. The overall path loss is expressed as the ratio between the radiated and received powers, thus from (5)

$$
\frac{P_{R A D}}{P_{r}}=d^{n}\left(\frac{4 \pi}{\lambda}\right)^{2}
$$

that can be written in $\mathrm{dB}$ form as

$$
L=10 n \log (d)+K_{0} \mathrm{~dB}
$$

where

$$
K_{0}=20 \log \left(\frac{4 \pi}{\lambda}\right) \mathrm{dB}
$$

is the attenuation (path loss) with $1 \mathrm{~m}$ between the antennae. From (6) it is clear that $n$ is also a random variable. If, in equation (8), the mean value of $n$ is used, then an additional random term is required, and equation (9) takes the form

$$
L=10 n \log (d)+K_{0}+X_{1} \mathrm{~dB}
$$

which is the usual form found in the literature [13-15], where $X_{1}$ is a random variable with zero mean that describes the statistical behaviour of the path loss $1 \mathrm{~m}$ from the transmitter.

The model proposed here is simply that given by equation (8) and the reason for not using the model given by (10) is that, in the measurements performed, it was found that the variations of the received power were considerably higher in the vicinity of the transmitting antenna, up to distances of the order of $20 \lambda$. Such behaviour, which cannot be explained only in terms of near-field effects, does not reflect the actual behaviour of the power, or in this case, of the exponent in large areas, as will be discussed in section 4. Independently of such a reason, the model given by equation (8) provides all necessary information regarding the path loss in the propagation channel and the values of $n$ can easily be obtained from experimental data by means of the following expression:

$$
n=\frac{P_{R A D}-P_{r}-K_{0}}{10 \log (d)}
$$


where $P_{r}$ are the measured samples of the received power. Furthermore, the excess attenuation referred to free space can be calculated as

$$
L_{0 E X}=10(n-2) \log (d) \mathrm{dB} .
$$

Application of equation (8) does not require any additional assumption, either from the physical or from the statistical point of view, or the introduction of any additional variable.

\section{Experimental details}

\subsection{Previous experimental considerations}

In order to calculate $n$ for each sample of received power, equation (8) must be used. However, it must be taken into account that $P_{r}$ is the isotropic power before the receiving antenna and not that measured by the instrument which, in our case, corresponds to the actual power at the input connector of a spectrum analyser. In a similar manner, the radiated power, $P_{R A D}$ is that radiated by the transmitting antenna and not that measurable at the transmitter output. Additional losses introduced by transmission lines as well as antenna gains both at the transmitter and at the receiver must also be considered. Thus, it is necessary to modify equation (11) in order to calculate $n$ in the following form:

$$
\langle n\rangle=\frac{P_{T}-\left\langle P_{R X}\right\rangle-K_{0}+K_{S}}{10 \log (d)}
$$

where $P_{T}$ is the transmitter output power, $P_{R X}$ the input power to the receiver and

$$
K_{S}=G_{T}+G_{R}-\sum L_{T}-\sum L_{R}
$$

expresses the fixed gains and losses at the transmitting (T) and receiving (R) ends, which are not dependent on propagation phenomena. It is important to notice that $G_{T}$ and $G_{R}$ are the nominal antenna gains, rather than the actual ones which, at each receiving point, can vary as a result of antenna coupling with nearby walls, objects or people. Those variations are assumed, from the model point of view, as propagation dependent and embedded within the value of $n$. Equation (13) allows one to calculate $n$ in terms only of measurable parameters.

\subsection{Experimental procedures}

Indoor propagation experiments were performed at $1.8 \mathrm{GHz}$ using a signal generator (HP8616A) as a transmitter and, as a receiver, a spectrum analyser (HP8569), computer controlled and mounted on a movable car, as shown in figure 1. The signal transmitted was an unmodulated carrier and the transmitting and receiving antennae were $\lambda / 4$ monopoles above ground. For the receiver, also a rotatory $\lambda / 2$ dipole was used to measure the co-polar and cross polar components in some of the experiments. The transmitting antenna was mounted at the top of a $2 \mathrm{~m}$ mast, and was kept fixed for each set of measurements. The reason for choosing this height was somewhat arbitrary; assuming a fixed transmitting station, such a height corresponds roughly to one half of the full height of most of the spaces where measurements were performed. The receiving antenna, at the top of a $1.5 \mathrm{~m}$ mast, was mounted on a platform fixed onto a $1.2 \mathrm{~m}$ rail with a calibrated scale of $1 \mathrm{~m}$ length, so the antenna could be displaced horizontally at fixed intervals. Also the rail was displaced at fixed intervals of $2.5 \mathrm{~cm}$ after each set of horizontal measurements.

The power output of the signal generator was adjusted to $10 \mathrm{dBm}$ at the beginning of each session with a HP432B power meter and the frequency was measured with a frequency counter. The transmitter and receiver were directly connected to the antennae with $\frac{1}{4}^{\prime \prime}$ co-axial cable. The losses of each cable, as well as the voltage standingwave ratio (VSWR) of the antennae were measured in the laboratory in order to calculate $K_{S}$ in equation (14).

Experiments were performed in two buildings of the University of Cantabria. One of them was of simple geometry with open areas and straight aisles. The other one was of more complex geometry, with narrow aisles that were occasionally curved and with numerous obstructions by walls, columns and furniture. The areas for the experiments were chosen in order to study the behaviour of the exponent of the distance under several conditions: line of sight (LOS), non-line of sight (NLOS), mainly shadowing and total obstruction between transmitter and receiver (OBS). The effect of random human traffic in the propagation environment was measured in the first building and, in the second, measurements of received signals under co-polar and cross polar conditions were also performed.

The measurement procedure was devised in order to make it easily applicable in practice and even though, in some of the experiments, data were recorded directly with a PC, in most of them the recording was made visually, having in mind the usual limitations in engineering practice in which it is not always possible to have to hand automatic or sophisticated equipment. This procedure introduces a mean error in the order of $\pm 1 \mathrm{~dB}$ in the visually recorded values, compared with those recorded automatically. In order to perform reliable measurements, two important considerations must be made, one dealing with the measurement interval, the other with the required number of samples. Regarding the measurement interval, the criteria adopted were to keep a minimum separation of $20 \lambda$ between local areas and a distance of $2.5 \mathrm{~cm}$ between samples in a local area $[17,18]$.

Regarding the number of samples, to describe the random variable statistically, in this case the exponent of the distance, the main parameters were the mean and standard deviation, so the number of samples in a local area should produce these parameters to reasonable approximations. The problem is then to establish the minimum reliable number of samples that must be taken in a local area. With such a purpose, various measurements were performed at different points of the same area, taking first 500 samples, then two sets of 100 and finally four sets of 50 samples each in four different local areas and under LOS and NLOS conditions. The maximum observed RMS error in the path loss between the means of the smaller sets, with respect to the mean of the largest sets, was of the order of $10^{-3}$ with $7 \times 10^{-2}$ for the standard deviation. Further measurements were performed taking a minimum of 40 samples with 


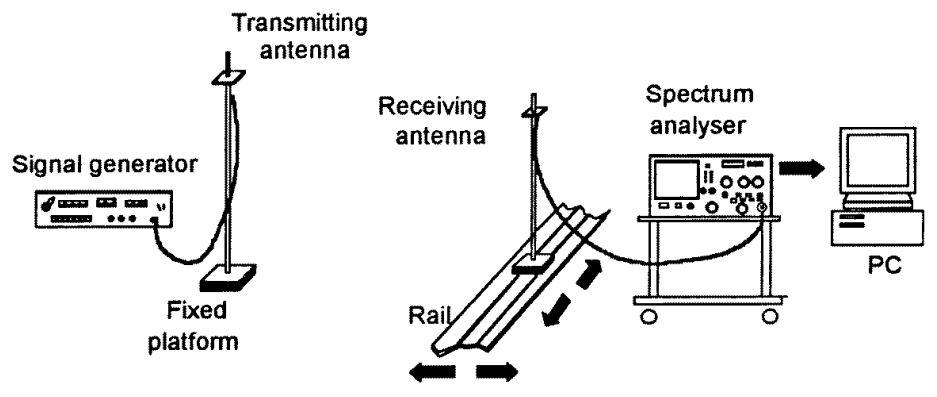

Figure 1. The experimental set-up.

errors in the mean value of the path loss of the order of $1.6 \times 10^{-2}$ with 0.7 for the standard deviation. Such results are in good agreement with the criteria suggested by Lee [17] and with those obtained with a purely theoretical approach [19]. For such reasons a minimum of 50 samples was considered adequate here.

However, it must be taken into account that, since the mean of the exponent can be assumed constant for a given propagation condition (LOS, NLOS, and so on) in a particular environment and excluding the region near the transmitter $(<20 \lambda)$, the various sets of data taken in local areas can be grouped for that specific condition, thus producing a larger set whose mean and standard deviation will be very close to the true ones. This is, in fact, an empirical application of the central-limit theorem. Such grouping is not possible if the variable is other than $n$, for instance the attenuation, power or electric field intensity.

Measurements were performed in corridors and open areas in both buildings under LOS conditions, at distances in the range 1-32 m. NLOS (shadowing) experiments were conducted along perpendicular corridors and semiobstructed open areas at distances of up to $25 \mathrm{~m}$. OBS measurements were carried out between different rooms and floors at distances of up to $22 \mathrm{~m}$. The effect of people in random movement in the propagation environment was measured in open areas with highly variable human traffic and finally, the effect of de-polarization was measured under the various propagation conditions.

\section{Results and their discussion}

Typical mean values and standard deviations of the exponent, observed for the various propagation conditions at distances $\geq 20 \lambda$ in the two buildings, are shown in table 1 .

\subsection{LOS}

The mean value of $n$ under LOS conditions was highly uniform in the different environments and close to its freespace value $(n=2)$. However, standard deviations differed and were highly dependent on the environment. In building 1 , where the aisles are wider and higher with several windows with metallic frames, mean standard deviation values in the range $0.337-0.54$ were observed and, as the distance between the transmitter and the receiver increased,

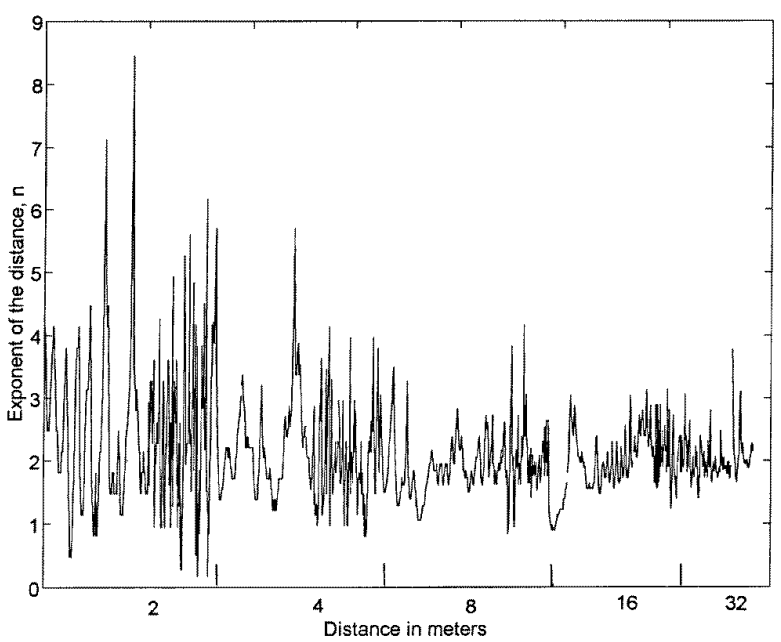

Figure 2. The profile of the exponent versus the distance under LOS conditions.

this parameter decreased slightly. Figure 2 shows a typical profile of the exponent in a LOS environment.

In building 2 , the aisles were narrow and lower. Measurements were performed at distances in the range 11-22 $\mathrm{m}$ and the observed values of the standard deviation $\sigma$ were more uniform and smaller than those in building 1 . For open areas in the two buildings, the observed standard deviations were similar.

\subsection{Behaviour at short distances}

At distances between the transmitter and the receiver of up to about $20 \lambda$, which must not be confused with the near field of the transmitting antenna, the behaviour of the exponent is highly variable. Its mean and standard deviation were higher than were those at larger distances. The same behaviour was observed under OBS conditions, even when the transmitter and receiver were separated by a wall. Under NLOS conditions, a similar behaviour occurred near the shadowing obstacle. Up to approximately $20 \lambda$, the mean of the exponent decreased approximately according to the following law:

$$
\bar{n}=a \overline{n_{0}}+b \mathrm{e}^{-k d}
$$

where $a, b$ and $k$ are constants dependent on the environment and $d$ is the distance between the transmitter 


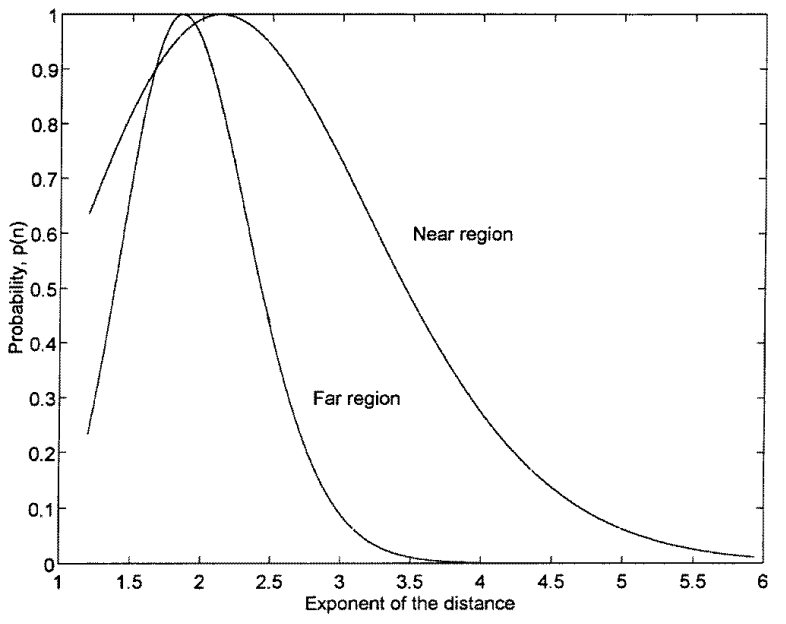

Figure 3. PDFs for the near and far regions under LOS conditions in the same environment.

and the receiver under LOS and OBS conditions and that between the shadowing obstacle and the receiver under NLOS conditions. Farther than 20 $\lambda$ the mean of the exponent reaches a nearly constant value. For the experiments reported here the following values were empirically found: $a \simeq 1.01, b$ in the range 1.4-1.6 and $k$ in the range $0.7-0.95$. As mentioned in section 2 , the variability of the exponent in this region was the reason for not using the model given by equation (10).

From a practical point of view, if the samples in the region at distances of the order of $20 \lambda$ or less are taken into account, the result will be a much more pessimistic prediction of the path loss, because of the higher means and standard deviations, as can be seen in figure 3 , in which probability density functions (PDF), normalized with respect to the maximum, are plotted for LOS conditions. Even when the mean values are very similar, the PDF for the samples in the near region $(<20 \lambda)$ is wider than that of the samples at larger distances. If the fading margin predicted by these samples is used in the calculation of the power budget of a system, the resulting radiated power will be higher than that predicted using only the samples at larger distances, an approach that seems more realistic, since, at short distances from the transmitter, the received power level will be high enough to ensure good reception even with large fading depths.

\subsection{NLOS}

Mean values of $n$ under NLOS conditions are not uniform and depend highly on the geometry and objects in the environment, as can be seen in figure 4 , in which the cumulative distributions for the values observed in one of the buildings, in which $n$ was in the range 2.6-3.79, are shown. The former was in a laboratory with a great amount of metallic furniture and equipment; and the latter was in an aisle partially obstructed by an open metallic door and furniture. Such differences in values suggest that, under NLOS conditions, no assumption concerning the value of $n$ can be made and it must be obtained through measurements.

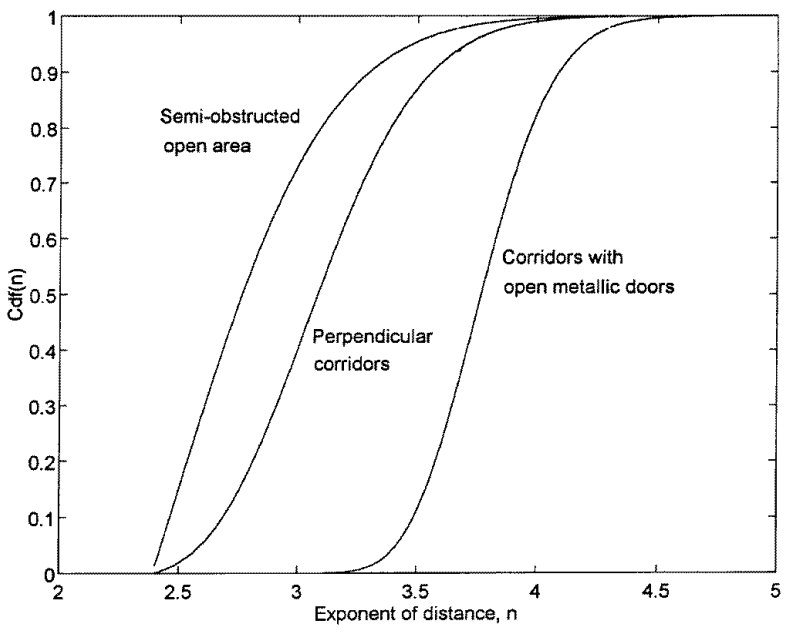

Figure 4. Cumulative distributions for the exponent under NLOS conditions in the same building.

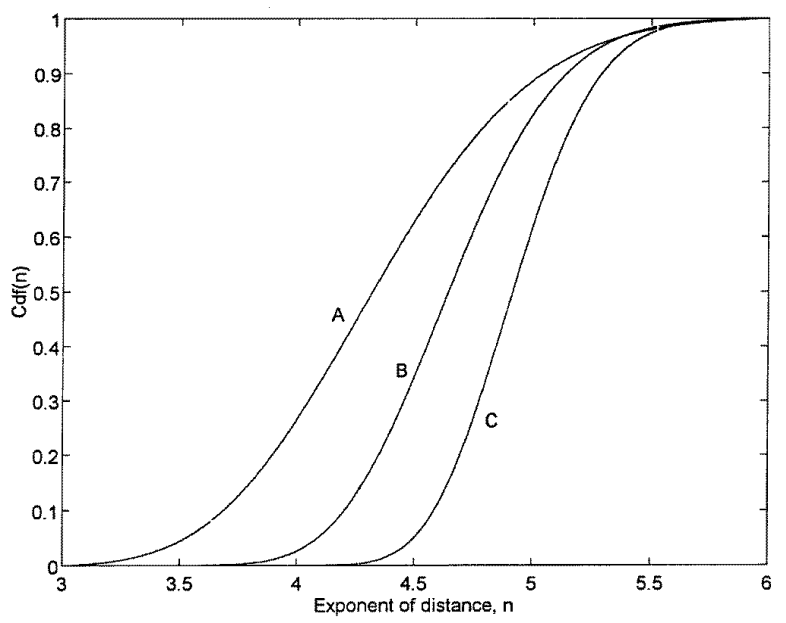

Figure 5. Effects of walls and complex obstacles in the same environment.

\subsection{OBS}

When obstacles in the propagation path are clearly defined equation (8) can be more properly expressed as

$$
L_{O B S}=10 n_{0} \log (d)+K_{0}+L_{W}
$$

where $n_{0}$ is the exponent measured in the region without the obstacle, $L_{W}$ represents the total fixed attenuation introduced by walls, floors or both and $d$, as before, is the distance between the transmitting and receiving antennae. In fact, for a mobile receiver or transmitter, $L_{W}$ is not constant and could also be assumed a random variable. However, if $L_{W}$ is taken to be approximately constant, the mean value of $n$ in the region after the obstacle will depend on the distance and can be expressed as

$$
\overline{n_{O B S}}(d)=\overline{n_{0}}+\frac{\overline{L_{W}}}{10 \log (d)} .
$$

Equation (17) provides a simple way to calculate the mean attenuation due to obstacles, $L_{W}$, via

$$
L_{W}=10\left[n_{O B S}\left(d_{0}\right)-n_{0}\right] \log \left(d_{0}\right)
$$


Table 1. Means and standard deviations $\sigma$ of the exponent observed in buildings 1 and 2 under various propagation conditions for distances $\geq 20 \lambda$.

\begin{tabular}{llllll}
\hline & \multicolumn{3}{c}{ Building 1 } & & \multicolumn{2}{c}{ Building 2 } \\
\cline { 2 - 3 } \cline { 5 - 6 } Case & $n_{\text {mean }}$ & $\sigma_{n}$ & & $n_{\text {mean }}$ & $\sigma_{n}$ \\
\hline LOS, aisles & 1.96 & 0.453 & & 1.97 & 0.187 \\
LOS, open area & 2.05 & 0.535 & & 2.07 & 0.528 \\
NLOS & 3.1 & 0.347 & & $2.6-3.79$ & $0.235-0.45$ \\
OBS, walls & 2.54 & 0.53 & & $4.35-4.93$ & $0.28-0.54$ \\
OBS, floors & & & & & \\
Human traffic & & & & 0.286 \\
LOS, low & $2.19-2.35$ & 0.155 & & \\
LOS, high & 2.47 & 0.344 & & \\
NLOS, variable & $2.36-2.64$ & $0.185-0.312$ & & \\
\hline
\end{tabular}

Table 2. Total attenuation factors.

\begin{tabular}{lcl}
\hline Obstacle & $L_{W}(\mathrm{~dB})$ & $\sigma_{L}(\mathrm{~dB})$ \\
\hline One wall & 5.75 & 0.75 \\
Three walls plus furniture (A) & 22.58 & 5.028 \\
Four walls plus furniture and some stairs (B) & 28.91 & 3.915 \\
Five walls plus furniture and the bulk of the stairs (C) & 34.69 & 3.216 \\
\hline
\end{tabular}

where $d_{0}$ is the distance between the transmitter and the receiver at which $n_{O B S}$ is measured.

The largest values of the exponent, $n_{O B S}$, occur at short distances from the obstacle and, since the attenuation introduced by it is constant, the exponent after the obstacle will depend on the distance, according to equation (17). Total attenuation factors were calculated with (18) for the various environments and their values are shown in table 2 (see figure 5).

However, the mean value of the exponent or the path loss is not sufficient, since it will give an indication of the $50 \%$ probability of the path loss not exceeding a certain value and provides no information about the necessary fading margin to ensure reliable reception. Therefore, measurements must be performed under the worst conditions for which reception has to be ensured, in order to estimate the adequate fading margin in a particular design.

\subsection{Human traffic}

To measure the effect of random human traffic on the total path loss, a series of measurements was conducted in the cafeteria of building 1 at different hours when the flow of people was moderate or high. In this case, the receiver was placed at a fixed site in different places of the cafeteria and samples were taken at $10 \mathrm{~s}$ intervals. In the absence of people and under LOS conditions, the value of the exponent was 2.19; with moderate traffic its value was 2.35 and with a high density of people, 2.47. Such values result in an excess attenuation, for the worst case, of $3.86 \mathrm{~dB}$. A value of $3.48 \mathrm{~dB}$ was calculated for NLOS conditions, under which the value of the exponent was in the range 2.36-2.64. The values observed in the experiments were lower than those reported by Karlsson [20], at $1.7 \mathrm{GHz}$, at which the attenuation due to body obstruction was in the range $6-8 \mathrm{~dB}$, the former in aisles and the latter in open areas. The differences from the values observed here were probably due to the fact that, in our experiments, the antennae were located at distances greater than $1.5 \mathrm{~m}$ from people for the receiver and about $4 \mathrm{~m}$ for the transmitter, whereas in [20], at least one of the antennae was close to a human head.

\subsection{Polarization effects}

Polarization effects were measured by taking two measurements at the same point, one with the receiving antenna vertically polarized and the second with horizontal polarization. The transmitting antenna was vertically polarized in all cases. Under LOS conditions, the ratio between the co-polar and the cross polar power had a mean value of $15.6 \mathrm{~dB}$. The cross polar power exceeded the copolar one only in 12 of 814 measurements. Under NLOS conditions, namely shadowing without total obstruction, that ratio was $6.57 \mathrm{~dB}$ and, for a total of 67 of 500 samples $(13.4 \%)$, the cross polar power exceeded the co-polar value. Under OBS conditions, the ratio was between 6.6 and $1.4 \mathrm{~dB}$ for the worst case, there being $34 \%$ of 300 samples for which the cross polar power was higher than the copolar one.

\subsection{The statistical behaviour}

The statistical behaviour of the exponent was analysed with several distribution functions, Rayleigh, Rice, log-normal, Weibull, Nakagami and gamma ones, for a total of nearly 70 sets of measurements. Very good fittings, in general, were obtained with the last three functions. Among them, the gamma distribution offered the best fit in the majority 
Table 3. Errors and correlation coefficients for a large area in corridors (NLOS).

\begin{tabular}{llllll}
\hline Function & $n_{\text {pmax }}$ & $\varepsilon_{\text {pdf }}$ & $\varepsilon_{c d f}$ & $c_{p d f}$ & $c_{c d f}$ \\
\hline Gamma & 3.043 & $2.34 \times 10^{-2}$ & $1.19 \times 10^{-2}$ & 0.9837 & 0.9997 \\
Nakagami & 3.085 & $2.16 \times 10^{-2}$ & $1.11 \times 10^{-2}$ & 0.9806 & 0.9996 \\
Weibull & 3.092 & $2.48 \times 10^{-2}$ & $1.13 \times 10^{-2}$ & 0.9806 & 0.9997 \\
\hline
\end{tabular}

Table 4. Errors and correlation coefficients for a local area (the worst case) in corridors (NLOS).

\begin{tabular}{llllll}
\hline Function & $n_{\text {pmax }}$ & $\varepsilon_{p d f}$ & $\varepsilon_{c d f}$ & $c_{p d f}$ & $c_{c d f}$ \\
\hline Gamma & 3.145 & $7.6 \times 10^{-2}$ & $4.35 \times 10^{-2}$ & 0.680 & 0.9954 \\
Nakagami & 3.179 & $7.88 \times 10^{-2}$ & $4.61 \times 10^{-2}$ & 0.6689 & 0.9944 \\
Weibull & 3.183 & $8.16 \times 10^{-2}$ & $4.36 \times 10^{-2}$ & 0.679 & 0.9953 \\
\hline
\end{tabular}

of cases, the Weibull and Nakagami functions being only marginally worse in many cases. The statistical behaviour of $n$ being known, the power budget of a system can be estimated for a given fading margin, namely the probability of not exceeding a certain path-loss value.

Two particular cases are shown in figures 6 and 7 . The former is for all the samples at distances greater than $20 \lambda$, in one corridor under NLOS conditions. In total 300 samples (50 for each local area) were grouped in one set which will be equivalent to the large-area description of the exponent. The mean value of the exponent was, for this set, 3.105 and the standard deviation was 0.347 . Fittings with gamma, Nakagami and Weibull distributions are almost indistinguishable. In figure 7, the worst fitting for a local area in the same corridor is shown. Here, only 50 samples were used, with a mean value of 3.188 and a standard deviation of 0.247 . Fitting differences are greater than those in the previous case; however, attention must be paid to the fact that the mean values and curve shapes are very similar.

The RMS errors between probability densities of samples and PDFs $\left(\varepsilon_{p d f}\right)$, cumulative distributions $\left(\varepsilon_{c d f}\right)$, correlation coefficients for the samples and theoretical PDFs $\left(c_{p d f}\right)$ as well as the corresponding CDFs $\left(c_{c d f}\right)$, are shown in tables 3 and 4 for the cases mentioned. The values at which the maximum probability occurs $\left(n_{\text {pmax }}\right)$ are also given in tables 3 and 4 .

The criteria by which to judge the quality of fit were based on the RMS errors between the sampling and theoretical probability density functions and cumulative distributions, and also on the correlation coefficients for relationships between the theoretical and real functions. The average errors between PDFs were of the order of $5 \times 10^{-2}$ and $2 \times 10^{-2}$ for the CDFs. The correlation coefficients were of the order of 0.91 for PDFs and 0.992 for CDFs.

\section{Conclusions}

A very simple power-law path-loss model, in which the exponent of the distance is the random variable, has been studied experimentally in different indoor environments. The exponent of the distance bears within it the effects of

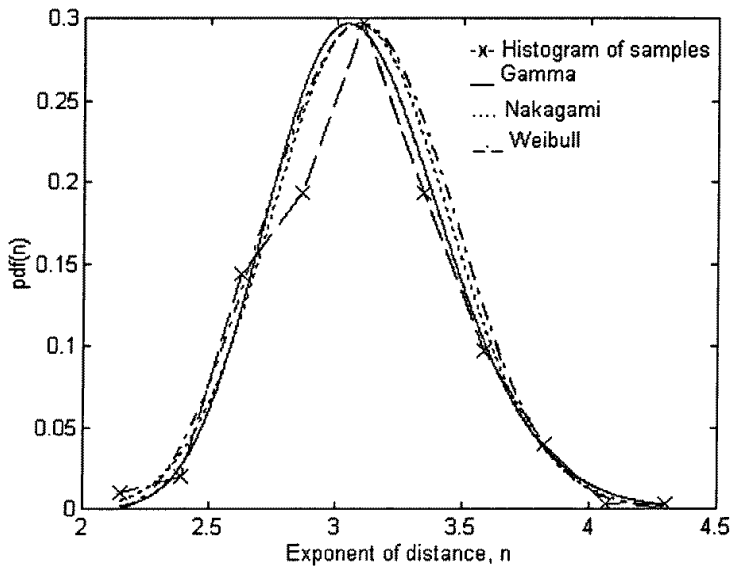

Figure 6. A comparison of fits to gamma, Nakagami and Weibull PDFs for a large area (300 samples), under NLOS conditions.

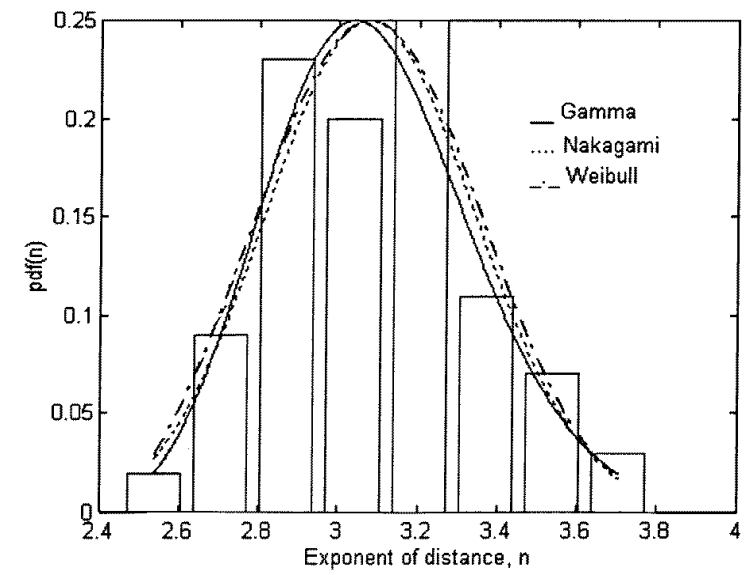

Figure 7. A comparison of fits to gamma, Nakagami and Weibull PDFs for a set of 50 samples in a local area under NLOS conditions.

the various propagation mechanisms that affect attenuation in the channel. The definition of such effects individually, from a physical point of view, is not required. The model can be applied in the estimation of the power budget of indoor wireless communication systems. However, 
it must be taken into account that the model does not provide a complete description of the propagation channel, for which the delay spread and phase behaviour must be included.

Even when the mean values of the exponent may be similar in different environments, the fading behaviour depends strongly on the topography. Such behaviour is reflected in the standard deviation and, for that reason, no assumptions can be made about its value. Also, under propagation conditions other than LOS, the mean value of the exponent may attain quite different values in the same building. From an engineering point of view, the worstcase conditions must be evaluated.

Electromagnetic energy is strongly de-polarized under conditions other than LOS. With heavy obstructions, the magnitudes of the co-polar and cross polar components are similar and human traffic in the environment can add about $4 \mathrm{~dB}$ to the total attenuation. Finally, very good fitting of the exponent values was obtained with Weibull, Nakagami and gamma distributions, the latter being better in general. On the basis of this proposed model, new, very simple and handy instruments can be developed for pathloss measurement and fading-margin estimation of indoor wireless communication systems.

\section{References}

[1] Keenan J M and Motley A J 1990 Radio coverage in buildings British Telecom. Technol. J. 8 19-24

[2] Motley A J and Keenan J M 1988 Personal communication radio coverage in buildings at $900 \mathrm{MHz}$ and $1700 \mathrm{MHz}$ Electron. Lett. 24 763-4

[3] Lafortune J F and Lecours M 1990 Measurement and modelling of propagation losses in a building at 900 MHz IEEE Trans. Vehic. Technol. 39 101-8

[4] Jimenez Delgado J et al 1993 Draft propagation model. European Co-operation in the Field of Scientific and Technical Research. COST231, TD(93)/19 Barcelona, January 1993
[5] Rappaport T S, Seidel S Y and Takamizawa K 1991 Statistical channel impulse response models for factory and open plan building radio communication system design IEEE Trans. Commun. 39 794-806

[6] Saleh A A M and Valenzuela R 1987 Statistical model for indoor multipath propagation IEEE J. Select. Areas Commun. 5 128-37

[7] Bello P A 1963 Characterisation of randomly time-variant linear channels IEEE Trans. Commun. Syst. 11 360-93

[8] Turin G L et al 1972 A statistical model of urban multipath propagation IEEE Trans. Vehic. Technol. 21 1-9

[9] Clarke H R 1968 A statistical theory of mobile-radio reception Bell Syst. Technical J. 47 957-1000

[10] Hashemi H 1993 The indoor radio propagation channel Proc. IEEE 81 943-67

[11] Lecours M et al 1996 Phase measurements and characterisation of mobile radio channels IEEE Trans. Veh. Technol. 45 105-13

[12] Suzuki H 1977 A statistical model for urban radio propagation. IEEE Trans. Commun. 25 673-80

[13] Rappaport T S 1996 Wireless Communications. Principles and Practice (New York: IEEE and Engelwood Cliffs, NJ: Prentice Hall PTR)

[14] Panjwani M A, Lynn Abbot A and Rappaport T 1996 Interactive computation of coverage regions for wireless communication in multifloored indoor environments IEEE J. Select. Areas Commun. 14 420-9

[15] Radiowave propagation document European Co-operation in the Field of Scientific and Technical Research. COST $231 \mathrm{TD}(93) / 13$ Barcelona January 1993

[16] Bach Andersen J, Rappaport T and Yoshida S 1995 Propagation measurements and models for wireless communications channels IEEE Commun. Mag. January $42-9$

[17] Lee W C 1985 Estimate of local average power of a mobile radio signal IEEE Trans. Vehic. Technol. 34 22-7

[18] Parsons J D 1992 The Mobile Radio Propagation Channel (London: Pentech)

[19] Kendall M and Stuart A 1977 The Advanced Theory of Statistics vol 1 and 2 4th edn (London: Charles Griffin)

[20] Karlsson P Body effects and diversity performance in indoor radio channels at $1700 \mathrm{MHz}$, European Co-operation in the Field of Scientific and Technical Research. COST231 TD(93)/14 Barcelona, January 1993 